\section{$\underset{\substack{\text { hommes } \\ \text { \& migrations }}}{ }$}

\section{Hommes \& migrations}

Revue française de référence sur les dynamiques

migratoires

$1326 \mid 2019$

Londres et ses migrations

\title{
Le Musée des civilisations noires a ouvert ses portes à Dakar
}

Mohamed Mbougar Sarr

\section{Q OpenEdition \\ Journals}

\section{Édition électronique}

URL : https://journals.openedition.org/hommesmigrations/9869

DOI : 10.4000/hommesmigrations.9869

ISSN : 2262-3353

Éditeur

Musée national de l'histoire de l'immigration

\section{Édition imprimée}

Date de publication : 1 juillet 2019

Pagination : 170-173

ISBN : 978-2-919040-46-9

ISSN : $1142-852 X$

\section{Référence électronique}

Mohamed Mbougar Sarr, "Le Musée des civilisations noires a ouvert ses portes à Dakar », Hommes \& migrations [En ligne], 1326 | 2019, mis en ligne le 01 juillet 2019, consulté le 16 janvier 2022. URL :

http://journals.openedition.org/hommesmigrations/9869; DOI : https://doi.org/10.4000/ hommesmigrations.9869 


\section{Initiatives Muséales}

\section{Le Musée des civilisations noires a ouvert ses portes à Dakar}

Mohamed Mbougar Sarr, écrivain, lauréat du Prix

littéraire de la Porte Dorée 2018.

Un demi-siècle après l'idée d'un musée consacré aux civilisations noires lancée par le poète Léopold Sédar Senghor, premier président du Sénégal, lors du premier Festival mondial des arts nègres en 1966, le Musée des civilisations noires (MCN) a été inauguré à Dakar, le 6 décembre 2018. Son architecture monumentale au cœur de la capitale sénégalaise, sa surface de $14000 \mathrm{~m}^{2}$ et son ambition d'accueillir à terme 18000 pièces, allant des vestiges archéologiques à l'art contemporain, font de ce nouveau musée une institution culturelle incontournable en Afrique de l'Ouest.

Je suis allé trois fois au Musée des Civilisations Noires entre fin mars et fin avril 2019: la première fois pour écouter un colloque qui s'y tenait, les fois suivantes pour voir ses collections. Et, à chacune de ces visites, la même scène s'est rejouée en amont: le chauffeur de taxi auquel je demandais de m'y emmener se montrait un peu hésitant sur la localisation du musée; l'un d'eux m'a même demandé ce que c'était. Toujours, il a fallu que je détaille, que je précise, que je fasse référence à l'imposant bâtiment circulaire inauguré en grande pompe par le chef de l'État début décembre 2018, avant que le lieu se dessine dans l'évidence d'un repère retrouvé: "Aaaaah, le gros bâtiment-là, à côté du Grand Théâtre, bien sûr, je vois... » Tel est, m'a-t-il semblé, le premier paradoxe du Musée des civilisations noires, qui vient d'ouvrir ses portes dans la capitale sénégalaise: s'il est difficile - vu ses dimensions, sa situation centrale dans la ville, le faste qui a accompagné son ouverture - de le rater, on peut avancer avec certitude qu'il faudra encore un peu de temps avant que les populations l'intègrent à leurs habitudes, leurs trajets, leurs stations, leurs repères géographiques et mentaux. Le nouveau venu dans le paysage des institutions culturelles se cherche doucement sa place dans la ville, dans l'esprit de ses habitants - peut-être dans leur cœur.

Il y a bien longtemps, pourtant, qu'on lui a promis cette place dans le paysage urbain et l'histoire du Sénégal: depuis plus de cinquante ans, puisque l'idée d'un musée négro-africain avait déjà été émise par Léopold Sédar Senghor lors du premier Festival mondial des arts nègres de 1966. Le projet, malgré le soutien de nombre d'experts et celui de l'Unesco, a dû être abandonné, la faute, en grande partie, aux funestement célèbres plans d'ajustement structurels imposés par le Fonds monétaire international (FMI) à nombre de pays africains pendant les années 1980 et 1990, sortes de feuilles de route économiques austères et restrictives, dans lesquelles la culture héritait de la portion congrue. Pendant ces longues années de désert culturel, pourtant, d'autres espaces, comme le Musée dynamique, ont continué à incarner des sortes d'esquisses ou de promesses renouvelées du Musée des civilisations noires. Le voilà donc aujourd'hui, au bout d'une longue épopée qui s'enracine dans l'histoire de la négritude et du panafricanisme.

Qu'aurait pensé Senghor devant son rêve concrétisé? Peut-être aurait-il salué cette monumentale construction comme symbole du dialogue des cultures: son architecture tient des cases traditionnelles casamançaises par la forme arrondie de ses flancs et des temples grecs par ses hautes colonnes (doriques? corinthiennes? ioniques? je ne sais plus; mais lui, distingué helléniste, aurait su). Sa construction a été financée - 30,5 millions d'euros - par le gouvernement chinois dans une logique active de partenariat politique (je ne veux pas savoir la nature de la contrepartie). Son ambition est de célébrer les civilisations noires et leurs apports à la civilisation universelle. Oui, Senghor aurait certainement été ému, et reconnaissant à son vieil adversaire politique, Abdoulaye Wade, d'avoir relancé en 2011 le projet que le successeur de ce dernier à la tête du pays, Macky Sall, a parachevé.

\section{Un contexte politique agité}

À ce propos, l'inauguration de l'endroit - «un peu précipitée pour des raisons politiciennes, car il n'est qu'à moitié prêt», me dit, dans ce que je crois être un euphémisme acide, un acteur influent du milieu culturel dakarois - a-elle obéi à un agenda politique national (les élections présidentielles eurent lieu trois mois après l'ouverture) ou s'inscrivait-elle dans un contexte politique plus large? Au Musée, on insiste sur la deuxième explication. Le lieu ouvre 
alors même que les débats sur les restitutions des œuvres d'art africaines, menés tambour battant par Bénédicte Savoy et Felwine Sarr"11, font rage dans les grandes institutions muséales européennes. Le Musée, lui, se tient prêt. À écouter Hamady Bocoum, son directeur, je comprends que l'existence même d'un tel espace, hypermoderne, constitue déjà un signal institutionnel clair à ceux qui douteraient de la capacité des pays africains à accueillir et à conserver leurs objets - qu'ils conservaient d'ailleurs parfaitement avant qu'on ne les leur enlève. Dans ce moment idéologique et historique important, donc agité, autour de la question du retour du patrimoine pillé en Afrique et gardé en Europe, le Musée des civilisations noires souhaite être un acteur politique et culturel majeur d'une relation renouvelée aux anciennes puissances esclavagistes et coloniales.

Se réapproprier et mettre en valeur les biens culturels qui faciliteront le travail sur l'estime de soi; réunir, par-delà les différentes expériences de fragmentation que furent l'esclavage et ses déportations, la colonisation et ses frontières, les migrations anciennes et récentes, les mondes noirs à travers leurs dénominateurs culturels; être non un Musée du ressentiment ou de la nostalgie, mais bien un espace où les cultures noires, pacifiées, seraient dans un dialogue fécond entre elles mais aussi avec les autres cultures: voilà les trois vocations majeures du musée.

\section{Refonder un imaginaire humilié}

Refondation d'un imaginaire humilié. Unification symbolique d'un grand espace culturel émietté. Travail d'apaisement de la mémoire et ouverture sur un dialogue contemporain entre civilisations égales en dignité. C'est du lourd. Un musée peutil, seul, prendre en charge une si grande tâche? Non, mais il doit prendre sa part, confie Hamady Bocoum. Comment? Le colloque auquel j'ai assisté lors de ma première visite au musée, intitulé «Patrimoine matériel, immatériel et altérité», me répond en partie. Felwine Sarr et Bénédicte Savoy en étaient les organisateurs. Que ce lieu abritât un tel événement m'apprenait deux choses. D'abord, et Hamady Bocoum l'a confirmé, que c'était aussi là sa vocation: être un espace où la recherche

1. Voir Felwine Sarr, Bénédicte Savoy, Restituer le patrimoine africain, Paris, Philippe Rey/Seuil, 2018.

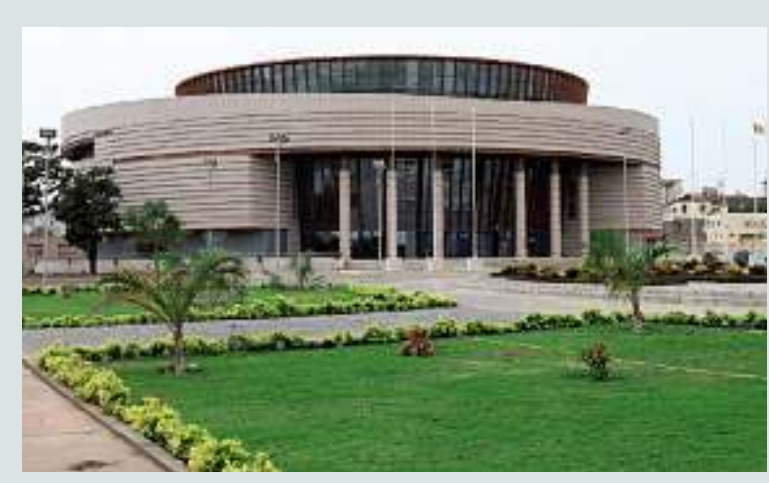

- Musée des civilisations noires, Dakar, 2019.

(C) Boubacar Konaté, MCN.

universitaire et la réflexion théorique seraient régulièrement en prise directe avec les collections, les objets, et les enjeux stratégiques, pratiques, que leur conservation et leur monstration soulevaient hic et nunc. Deuxième leçon: ce sont les questions mêmes des formes, des devenirs et des fonctions politiques des musées dans les «épistémologies des Suds», actuellement marquées par un mouvement décolonial fort et de plus en plus radical, que cet endroit pose.

Je ne saurai pas vraiment la position officielle du Musée sur la question des restitutions et/ou des modalités du retour des œuvres africaines vers leur continent d'origine (définitif? provisoire? total? partiel ?). C'est que cette position n'est pas tranchée: si on salue la solidité du rapport Sarr/ Savoy et le courage de ses recommandations, la sensibilité du sujet, la lenteur des protocoles (il reste encore, dans le cas du Sénégal, certains inventaires à dresser), ajoutées à la jeunesse du Musée, imposent, pour l'heure, une relative souplesse. Hamady Bocoum n'est pas une personnalité de rupture: sa conception du sens de ce Musée n'est pas celle d'un repli, mais d'une ouverture sur l'avenir, par la collaboration et la solidarité entre institutions culturelles. Dans un premier temps, le Musée n'aura d'ailleurs pas de collection permanente, et fonctionnera sous le modèle des partenariats, via des accords noués avec plusieurs musées internationaux. La constitution d'une collection permanente se fera progressivement; elle est même une ambition assumée dans le court terme, avec une volonté d'acquérir en priorité des œuvres d'artistes africains (du continent et de la diaspora) contemporains. 


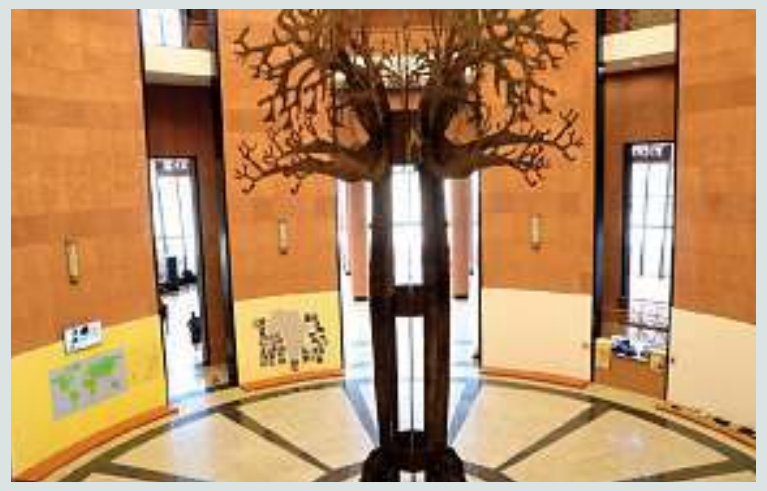

- «Baobab», artiste Edouard Duval-Carré, 2019.

(c) Boubacar Konaté, MCN.

\section{L'expérience de visite}

Mais allons-y voir de plus près (après avoir payé le prix du ticket d'entrée, bien sûr: 2000 francs CFA, environ 3 euros, ce qui me semble d'abord être une somme modique, avant que je ne me souvienne qu'il y a dans ce pays beaucoup de gens qui vivent avec moins que cela par jour).

Le grand vestibule, au centre duquel se dresse, comme une colonne symbolique centrale, une immense sculpture de baobab (œuvre de l'artiste haïtien Édouard Duval-Carrié), montre de grandes affiches où s'énoncent les raisons d'être et les objectifs du musée, ainsi que les propositions de parcours. L'exposition inaugurale s'intitule «Civilisations africaines: création continue de l'humanité». Elle s'étend sur quatre étages qui correspondent chacun à un thème du parcours: «le Berceau de l'humanité», «les Civilisations africaines continentales», «la Mondialisation de la Négritude», «Maintenant l'Afrique». Ce découpage thématique - mais aussi, par de nombreux aspects, chronologique - permet de remonter à la genèse de notre espèce en Afrique (émouvante reconstitution des crânes et ossature de "Toumaï» et «Dinknesh/ Lucy», précurseurs glorieux de l'humanité, exhumés de la Rift Valley et du désert tchadien), avec une attention marquée pour la période de l'apparition du fer, avant d'en arriver aux manifestations les plus contemporaines des cultures noires, en passant par la formation des premiers foyers de civilisations sur le continent et leur expansion (parfois violente, traumatique et meurtrière) à travers le monde.

Une phrase du catalogue de présentation de cette exposition d'ouverture retient mon attention; elle répond - théoriquement, du moins - à des questions que je me suis posées dès que j'ai entendu l'appellation de "civilisations noires»: qu'entend-on par là? quelles sont les bornes thématiques et chronologiques d'un tel musée? La phrase est la suivante: «...l'exposition inaugurale constitue donc un processus d'articulation - d'une Afrique imaginée s'étendant au-delà de ses frontières continentales, incluant les civilisations africaines nées des traites esclavagistes transatlantiques "Passage du Milieu", transsaharien, et à travers l'Océan Indien -; soulignant ainsi la continuité au sein d'un processus qui réexamine le passé à la lumière de nouvelles configurations». Je perçois à peu près l'idée, même si l'adjectif «imaginée» m'a laissé un temps perplexe, moins sûr de ce qu'il fallait comprendre. Je me demande aussi pourquoi est-ce que le mot «diaspora» apparaît si peu pendant le parcours alors qu'on fait sans cesse référence à sa réalité.

Quant au détail de la visite elle-même, chacun s'en fera un avis. Je dois quand même dire, pour ma part, que passée la première séquence sur l'origine des hominidés («Le Berceau de l'humanité»), extrêmement bien documentée et renseignée, les étages suivants m'ont parfois un peu perdu, d'abord littéralement (j'ai eu du mal à m'orienter dans le parcours, que j'ai régulièrement pris à contresens, mais sans doute est-ce parce que j'ai une faculté d'orientation médiocre). J'aurais peutêtre dû, humblement, demander mon chemin à l'un des étudiants (en histoire de l'art, anthropologie, archéologie ou histoire) qui pouvaient guider ou renseigner les visiteurs, et que le Musée contribue ainsi - ce qui est une excellente chose - à former en leur offrant la possibilité pratique de prolonger leurs cours théoriques. Perdu, je l'ai ensuite été par les propos et les contenus, que j'ai trouvés parfois un peu confus ou elliptiques. Certaines images ne sont pas commentées ou renseignées par des cartels suffisamment détaillés (si cartel il y a). Résultat: il peut arriver que l'on croise un mur de personnalités historiques noires, une sorte de black hall of fame dont la logique de voisinage (autre que celle de leur communauté de couleur) interroge, si tant est qu'on les identifie d'abord toutes. Ce qui n'est pas toujours évident sans commentaire, quand bien même ils et elles seraient nos frères et sœurs de «couleur». Il faut croire que ça ne suffit pas. Bon. 


\section{Un espace en devenir}

Cependant, la critique principale que je pourrais faire à l'exposition, à l'issue de mes deux visites, est l'impression de grand «vide» spatial, qui ne m'a pas quitté tout le long de mon exploration. Il y a encore trop peu d'œuvres (pour cette exposition du moins), et on est loin des 18000 objets que l'espace affirme vouloir accueillir. Hamady Bocoum évoque de futures collaborations entre musées africains, la constitution d'un réseau au sein duquel le Musée des civilisations noires jouerait un rôle central, et le fait que des objets en provenance d'autres pays du continent étaient attendus, pour confirmer la vocation panafricaine des lieux. Je me suis demandé pour quelle raison certaines collections d'objets du Musée Théodore Monod de l'Ifan, magnifiques, n'étaient pas déplacées dans le Musée des civilisations noires. Elles y auraient largement leur place et y seraient peut-être plus mises en valeur qu'au Musée Monod, très riche, mais dont les structures m'avaient semblé un peu vétustes lors de ma dernière visite. Peut-être que la question est en train d'être étudiée. En attendant, les quelque $14000 \mathrm{~m}^{2}$ du Musée des civilisations semblent un peu grands, et les objets présents y nagent parfois comme des poissons dans un aquarium trop large.

Cela donne-t-il raison à la personne qui raillait l'ouverture précipitée du Musée pour satisfaire une commande politicienne? Je n'irai pas jusque-là. Il y a des choses perfectibles dans la scénographie, l'intelligence de l'espace, la contextualisation de certains objets rituels et la lisibilité des parcours, mais il y a déjà de belles pièces, un effort de cohérence dans la réflexion, et un souci d'ouverture pour rendre compte de la pluralité, au sein de ces civilisations noires, des cultures africaines ou afrodescendantes extracontinentales, presque toutes représentées - de l'océan Indien à l'Amérique du Sud. Les expositions de textiles sont magnifiques, et j'ai du reste eu grand plaisir à voir un pan entier de l'exposition consacrée à la Négritude (on y rend un bel hommage à la maison d'édition et à la revue éponyme Présence africaine, qui bénéficie d'ailleurs d'un espace dédié à la librairie du Musée, et à son fondateur, le grand Alioune Diop). L'espace du Musée, dans la lignée de la maison d'édition historique, entend être à la pointe des débats intellectuels brûlants sur les cultures noires et un lieu de promotion des arts africains. Votre serviteur

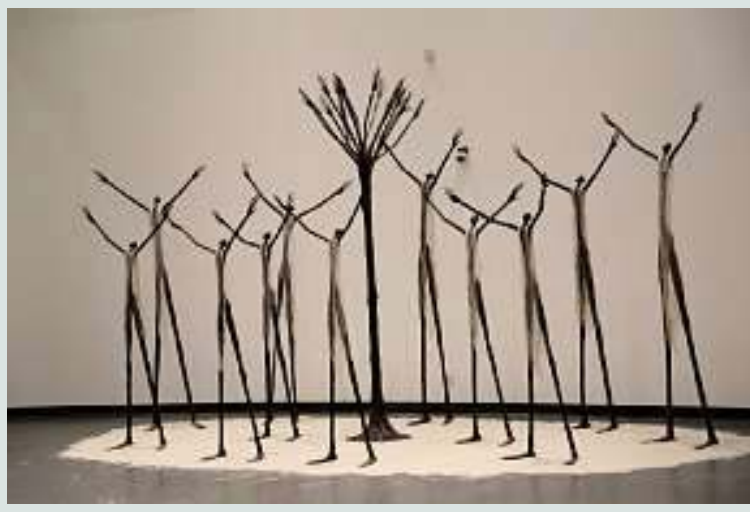

- Exposition, Musée des civilisations noires.

(c) Boubacar Konaté, MCN.

s'y est même vu proposer de participer à une table ronde sur une thématique liée à son premier roman et a pu assister à un débat vif sur les féminismes africains.

Reste une question, qui m'a accompagné tout le temps de mes visites: qui est là? Qui fréquente le Musée? Sans surprise, le public, pour l'heure, est majoritairement constitué de touristes occidentaux, ce qui est d'ailleurs tout à fait cohérent si on songe au discours du président de la République, Macky Sall, lors de l'inauguration, qui affirmait sans ambages vouloir aussi faire du Musée des civilisations noires un argument en faveur tourisme, un des fleurons de "l'émergence» dont il a fait l'obsession de ses deux mandats. Avec Gorée, Dakar dispose désormais de deux hauts lieux de mémoire autour de l'histoire des peuples noirs sur le continent et dans la diaspora. J'ai, en revanche, peu vu la population locale. Cela ne m'étonne pas, mais cela m'attriste. La proximité du Musée des civilisations noires avec le Grand Théâtre, lieu d'activités culturelles très couru par les Dakarois, notamment pour ses concerts, lui donne une certaine visibilité. Mais il pourrait aussi pâtir de la comparaison. L'un des défis de ce lieu sera également de travailler à résorber une sorte de fracture culturelle qui fait penser à certaines populations qu'une certaine dimension de la culture ne les intéresse ni les concerne. Seule consolation, à ce sujet, lors de ma dernière visite: la présence bruyante mais sympathique d'une classe d'enfants venue découvrir les lieux. L'un d'eux, devant le crâne de Toumaï, demande: c'est pour nous? C'est, au sujet de l'endroit, une merveilleuse question. 